\title{
ABSOLUTE CONTINUITY AND UNIQUENESS OF MEASURES ON METRIC SPACES
}

\author{
BY \\ PERTTI MATTILA
}

\begin{abstract}
We show that if two Borel regular measures on a separable metric space are in a suitable sense homogeneous, then they are mutually absolutely continuous. We use such absolute continuity theorems together with some density theorems to prove the uniqueness of measures more general than Haar measures.
\end{abstract}

1. Introduction. In this paper we prove absolute continuity and uniqueness theorems on a separable metric space $X$ for Borel regular measures which are homogeneous in the sense that the measures of the small balls of the same radii are roughly of the same magnitude. We shall consider two cases separately. In the first case we consider measures $\mu$ which satisfy

$$
\underset{r \downarrow 0}{\lim \sup } \frac{\mu B(x, 5 r)}{\mu B(x, r)}<\infty \quad \text { for } x \in X .
$$

(The number 5 comes from covering theorems, but obviously (1) holds if and only if it holds when 5 is replaced with some $t>1$.) In the second case we assume no $5 r$-condition, but then we must make stronger homogeneity assumptions. (For the necessity of these see Remark 4.8.) We call the first case finite dimensional and the second infinite dimensional because (1) holds for several interesting measures on finite-dimensional spaces, whereas it is false in typically infinite-dimensional cases.

Let $\mu_{1}$ and $\mu_{2}$ be Borel regular measures on $X$. In the absolute continuity theorems we prove that if there are nondecreasing functions $h_{i}:(0, \infty) \rightarrow(0, \infty)$ with $\lim _{r \downarrow 0} h_{i}(r)=0, i=1,2$, such that either

$$
0<\liminf _{r \downarrow 0} \frac{\mu_{i} B(x, r)}{h_{i}(r)}<\operatorname{lim\operatorname {sup}} \frac{\mu_{i} B(x, r)}{h_{i}(r)}<\infty
$$

and

$$
\underset{r \downarrow 0}{\lim \sup } h_{i}(5 r) / h_{i}(r)<\infty
$$

for $x \in X, i=1,2$, or

$$
0<\lim _{r \downarrow 0} \inf _{i} B(x, r) / h_{i}(r) \text { and } \mu_{i} B(x, r)<h_{i}(r)
$$

for $x \in X, r>0, i=1,2$, then $\mu_{1}$ and $\mu_{2}$ are mutually absolutely continuous. Our uniqueness theorems can be proved with the help of these absolute continuity

Received by the editors December 27, 1979 and, in revised form, May 13, 1980.

AMS (MOS) subject classifications (1970). Primary 28A10; Secondary 28 A70.

${ }^{1}$ Supported in part by NSF Grant MCS77-18723(02). 
theorems and some density theorems. It is possible to give somewhat shorter proofs to 3.3 and 4.3 which would rely on the general results of differentiation of integrals of $[3, \S 2.9]$ and [4], but I have preferred to keep the treatment self-contained in this respect. Some uniqueness theorems for measures which are invariant under certain families of homeomorphisms of $X$ follow as special cases, and they extend earlier results of Mickle and Radó [5] and Engle [2].

In the finite-dimensional case the proofs rely on the fact that from any family of closed balls of uniformly bounded radii one can select a countable disjoint subfamily such that the corresponding balls with five times larger radius cover the union of the original family. In the infinite-dimensional case the basic tool is Fubini's theorem.

To simplify the exposition we only consider measures on separable metric spaces, but similar methods could also be used to prove analogous results for measures on uniform spaces (cf. [4] where differentiation theorems are proved in uniform spaces).

\section{Preliminaries.}

2.1. Notation. Throughout the whole paper $(X, d)$ will be a separable metric space. For $x \in X$ and $r>0$ we denote by $B(x, r)$ the closed ball with centre $x$ and radius $r$. We let $H$ be the set of all nondecreasing functions $h:(0, \infty) \rightarrow(0, \infty)$ such that $\lim _{r \downarrow 0} h(r)=0$, and we denote

$$
H^{*}=\left\{h \in H: \limsup _{r \downarrow 0} h(5 r) / h(r)<\infty\right\} .
$$

Let $\mu$ be a Borel regular measure on $X$, that is, $\mu$ is an (outer) measure on $X$ such that all Borel sets are $\mu$ measurable, and for any $A \subset X$ there is a Borel set $B$ containing $A$ such that $\mu(A)=\mu(B)$. If $h \in H$ and $x \in X$, we define the lower and upper $h$-densities of $\mu$ at $x$ by

$$
\begin{aligned}
& \underline{D}_{h}(\mu, x)=\liminf _{r \downarrow 0} \mu B(x, r) / h(r), \\
& \bar{D}_{h}(\mu, x)=\limsup _{r \downarrow 0} \mu B(x, r) / h(r) .
\end{aligned}
$$

If they are equal, their common value

$$
D_{h}(\mu, x)=\lim _{r \downarrow 0} \mu B(x, r) / h(r)
$$

is the $h$-density of $\mu$ at $x$. The functions $\underline{D}_{h}(\mu$,$) and \bar{D}_{h}(\mu$,$) are Borel functions.$

For any $A \subset X$ the restriction measure $\mu \mathrm{L} A$ is defined by $(\mu \mathrm{L} A)(B)=\mu(A \cap B)$ for $B \subset X$.

If $E \subset X, \chi_{E}$ is the characteristic function of $E$.

The following two theorems will be useful.

2.2. TheOREM [3, 2.2.2-3]. Suppose that $\mu$ is a Borel regular measure on $X, B$ is a $\mu$ measurable set in $X$, and $\varepsilon>0$.

(1) If $\mu(B)<\infty$, then $B$ contains a closed set $C$ for which $\mu(B \backslash C)<\varepsilon$.

(2) If $B$ is contained in the union of countably many open sets $V_{i}$ with $\mu\left(V_{i}\right)<\infty$, then $B$ is contained in an open set $W$ for which $\mu(W \backslash B)<\varepsilon$. 
2.3. Theorem [3, 2.8.4-5]. If $\mathscr{B}$ is a family of closed balls of bounded radii in $X$, then $\mathscr{B}$ has a disjoint countable subfamily $\left\{B\left(x_{i}, r_{i}\right): i=1,2, \ldots\right\}$ such that $\cup \mathscr{B} \subset \cup_{i=1} B\left(x_{i}, 5 r_{i}\right)$.

\section{The finite-dimensional case.}

3.1. TheOREM. Let $\mu$ be a Borel regular measure on $X$ and $E$ a $\mu$ measurable subset of $X$. If $\mu B(x, r)<\infty$ for small $r$ for $\mu$ a.a. $x \in X$, then

$$
\lim _{r \downarrow 0} \frac{\mu(B(x, r) \backslash E)}{\mu B(x, 5 r)}=0 \text { for } \mu \text { a.a. } x \in E \text {. }
$$

Proof. Let $G$ be an open set with $\mu(G)<\infty$ and $F$ a closed subset of $G \cap E$. Since $X$ is separable, it is sufficient by $2.2(1)$ to show that the asserted equation holds $\mu$ a.e. in $F$. To do this let $\varepsilon>0$ and let $A_{\varepsilon}$ be the set of all $x \in F$ for which

$$
\lim \sup _{r \downarrow 0} \frac{\mu(B(x, r) \backslash E)}{\mu B(x, 5 r)}>\varepsilon .
$$

There is a closed set $C \subset G \backslash E$ such that $\mu(G \backslash E \backslash C)<\varepsilon^{2}$. For each $x \in A_{\varepsilon}$ we can find $0<r(x)<1$ such that $B(x, r(x)) \subset G \backslash C$ and $\mu(B(x, r(x)) \backslash E)>$ $\varepsilon \mu B(x, 5 r(x))$. By 2.3 there are $x_{i} \in A_{e}, i=1,2, \ldots$, such that the balls $B\left(x_{i}, r_{i}\right)$, where $r_{i}=r\left(x_{i}\right)$, are disjoint and the balls $B\left(x_{i}, 5 r_{i}\right)$ cover $A_{\varepsilon}$. Then

$$
\begin{aligned}
\mu\left(A_{\varepsilon}\right) & <\sum_{i=1}^{\infty} \mu B\left(x_{i}, 5 r_{i}\right)<\frac{1}{\varepsilon} \sum_{i=1}^{\infty} \mu\left(B\left(x_{i}, r_{i}\right) \backslash E\right) \\
& <\varepsilon^{-1} \mu(G \backslash E \backslash C)<\varepsilon .
\end{aligned}
$$

Hence $\mu\left(\cup_{e>0} A_{e}\right)=\lim _{e \downarrow 0} \mu\left(A_{\varepsilon}\right)=0$, from which the theorem follows.

3.2. COROLlaRY. If in addition to the assumptions of Theorem 3.1,

$$
\underset{r \downarrow 0}{\lim \sup } \frac{\mu B(x, 5 r)}{\mu B(x, r)}<\infty \quad \text { for } \mu \text { a.a. } x \in E,
$$

then

$$
\lim _{r \downarrow 0} \frac{\mu(B(x, r) \backslash E)}{\mu B(x, r)}=0 \text { and } \lim _{r \downarrow 0} \frac{\mu(E \cap B(x, r))}{\mu B(x, r)}=1
$$

for $\mu$ a.a. $x \in E$.

Proof. The first assertion follows upon writing

$$
\frac{\mu(B(x, r) \backslash E)}{\mu B(x, r)}=\frac{\mu(B(x, r) \backslash E)}{\mu B(x, 5 r)} \frac{\mu B(x, 5 r)}{\mu B(x, r)},
$$

and the second follows from the first.

3.3. ThEOREM. Suppose that $\mu$ and $\nu$ are Borel regular measures on $X, g \in H^{*}$ and $h \in H$. If for each $x \in X$ there exist $0<c_{x}<C_{x}<\infty$ such that

$$
\liminf _{r \downarrow 0} \frac{\mu B\left(x, C_{x} r\right)}{g(r)}>0, \quad \limsup _{r \downarrow 0} \frac{\mu B\left(x, c_{x} r\right)}{g(r)}<\infty
$$


and

$$
\liminf _{r \downarrow 0} \frac{\nu B\left(x, C_{x} r\right)}{h(r)}>0, \quad \lim \sup \frac{\nu B\left(x, c_{x} r\right)}{h(r)}<\infty
$$

then

$$
\lim _{r \downarrow 0} \inf g(r) / h(r)>0, \quad \lim \sup g(r) / h(r)<\infty
$$

and $\mu$ and $\nu$ are mutually absolutely continuous.

Proof. Choose an open set $G \subset X$ such that $\mu(G)<\infty$ and $\nu(G)<\infty$. We can find $0<c<1<C<\infty, 0<d<D<\infty, 0<r_{0}<1$ and a closed set $F \subset G$ such that $\mu(G) \leqslant 2 \mu(F)$, $\operatorname{dist}(F, X \backslash G)>r_{0}$ and

$$
d g(c r)<\mu B(x, r)<D g(C r), \quad d h(c r)<\nu B(x, r)<D h(C r)
$$

for all $x \in F$ and $0<r<r_{0}$. Since $g \in H^{*}$, there is $\lambda<\infty$ such that $g(5 C r)<$ $\lambda g(c r)$ for $0<r<1$. Given $0<r<r_{0} / 5$, there are, by 2.3 , disjoint balls $B\left(x_{i}, r\right) \subset$ $G, i \in I(r)$, such that $x_{i} \in F$ and the balls $B\left(x_{i}, 5 r\right)$ cover $F$. Since

$$
\sum_{i \in I(r)} \mu B\left(x_{i}, r\right)<\mu(G)<\infty \text { and } \mu B\left(x_{i}, r\right)>d g(c r) \text { for } i \in I(r),
$$

$I(r)$ is finite, say, $I(r)=\{1, \ldots, k(r)\}$. Then

$$
\begin{aligned}
k(r) d g(c r) & <\sum_{i=1}^{k(r)} \mu B\left(x_{i}, r\right)<\mu(G)<2 \mu(F) \\
& <2 \sum_{i=1}^{k(r)} \mu B\left(x_{i}, 5 r\right)<2 k(r) D g(5 C r)<2 k(r) D \lambda g(c r) .
\end{aligned}
$$

Hence for $0<r<c r_{0} / 5$,

$$
k(r / c) \operatorname{dg}(r)<\mu(G)<2 k(r / c) D \lambda g(r) .
$$

In the same way for $0<r<c r_{0} / 5$,

$$
k(r / c) d h(r)<\nu(G)<2 k(r / c) D h(5 C r / c) .
$$

Thus for $0<r<c r_{0} / 5$,

$$
h(r)<a g(r) \text { with } a=2 D \lambda \nu(G)(d \mu(G))^{-1}
$$

and

$$
g(r)<\lambda g(c r /(5 C))<b h(r) \text { with } b=2 D \lambda \mu(G)(d \nu(G))^{-1} .
$$

It follows that (3) holds.

Next we prove that $\nu$ is absolutely continuous with respect to $\mu$. It is sufficient to show that $\nu(A)=0$ whenever $A \subset F$ with $\mu(A)=0$, where $F$ is as above. Let $\varepsilon>0$. By 2.2(2) there is an open set $U$ such that $A \subset U$ and $\mu(U)<\varepsilon$. For each $x \in A$ there is $0<r<c r_{0} /(25 C)$ such that $B(x, r) \subset U$,

$$
\mu B(x, r)>d g(c r)>d \lambda^{-1} g(r) \text { and } \nu B(x, 5 r)<D h(5 C r) \text {. }
$$

By 2.3 there is a disjoint sequence $\left(B\left(x_{i}, r_{i}\right)\right)$ of such balls with

$$
A \subset \bigcup_{i=1}^{\infty} B\left(x_{i}, 5 r_{i}\right)
$$


Then

$$
\begin{aligned}
\nu(A) & \leqslant \sum_{i=1}^{\infty} \nu B\left(x_{i}, 5 r_{i}\right)<D \sum_{i=1}^{\infty} h\left(5 C r_{i}\right)<D a \sum_{i=1}^{\infty} g\left(5 C r_{i}\right) \\
& <D a \lambda \sum_{i=1}^{\infty} g\left(r_{i}\right)<D a \lambda^{2} d^{-1} \sum_{i=1}^{\infty} \mu B\left(x_{i}, r_{i}\right) \\
& \leqslant D a \lambda^{2} d^{-1} \mu(U)<D a \lambda^{2} d^{-1} \varepsilon .
\end{aligned}
$$

Since $\varepsilon>0$ was arbitrary, $\nu(A)=0$. In the same way one proves that $\mu$ is absolutely continuous with respect to $\nu$.

3.4. THEOREM. Let $\mu$ and $\nu$ be Borel regular measures on $X$. Suppose that there is $x_{0} \in X$ such that

$$
\underset{r \downarrow 0}{\lim \sup } \mu B\left(x_{0}, 5 r\right) / \mu B\left(x_{0}, r\right)<\infty,
$$

and that for all $x, y \in X, r>0$ there are $0<c_{x y}<C_{x y}<\infty, r_{x y}>0$, independent of $r$, and a Borel set $A(x, y, r)$ such that

$$
\begin{gathered}
B\left(y, c_{x, y} r\right) \subset A(x, y, r) \subset B\left(y, C_{x_{y}} r\right) \text { for } 0<r<r_{x, y}, \\
\underset{r \downarrow 0}{\lim \sup } \frac{\mu A\left(x_{0}, x, r\right)}{\mu B\left(x_{0}, r\right)}<\infty, \quad \liminf _{r \downarrow 0} \frac{\nu A\left(x_{0}, x, r\right)}{\nu B\left(x_{0}, r\right)}>0
\end{gathered}
$$

and

$$
\underset{r \downarrow 0}{\lim \sup } \frac{\nu A(x, y, r)}{\nu B(x, r)}<\liminf _{r \downarrow 0} \frac{\mu A(x, y, r)}{\mu B(x, r)} .
$$

Then there is a constant $c$ such that $\nu=c \mu$.

Proof. Define $g(r)=\mu B\left(x_{0}, r\right), h(r)=\nu B\left(x_{0}, r\right), c_{x}=c_{x_{0} x}$ and $C_{x}=C_{x_{0} x}$ for $r>0, x \in X$. Then the assumptions of Theorem 3.3 hold. Hence 3.3(3) holds and $\mu$ and $\nu$ are mutually absolutely continuous. These inequalities together with lim sup $\operatorname{su0}_{r \downarrow} g(5 r) / g(r)<\infty$ and inequalities 3.3(1) and 3.3(2) imply that

$$
\underset{r \downarrow 0}{\lim \sup } \frac{\mu B(x, 5 r)}{\mu B(x, r)}<\infty \text { and } \limsup _{r \downarrow 0} \frac{\nu B(x, 5 r)}{\nu B(x, r)}<\infty
$$

for all $x \in X$. Since $\mu$ and $\nu$ are Borel regular and mutually absolutely continuous, the Radon-Nikodým theorem yields a positive and finite Borel function $f$ on $X$ such that $\nu(A)=\int_{A} f d \mu$ for all Borel sets $A \subset X$. We shall show that $f$ equals a constant $\mu$ a.e. If this is not true, there are $0<s<t<\infty$ such that the sets $A=\{x: f(x)<s\}, B=\{x: f(x)>t\}$ have both positive $\mu$ and $\nu$ measure. Then by Corollary 3.2 there are $a \in A$ and $b \in B$ such that

$$
\lim _{r \downarrow 0} \frac{\nu(A \cap B(a, r))}{\nu B(a, r)}=1, \quad \lim _{r \downarrow 0} \frac{\mu(B(b, r) \backslash B)}{\mu B(b, r)}=0 .
$$

From

$$
\frac{\mu(A(a, b, r) \backslash B)}{\mu A(a, b, r)}<\frac{\mu\left(B\left(b, C_{a, b} r\right) \backslash B\right)}{\mu B\left(b, C_{a, b} r\right)} \cdot \frac{\mu B\left(b, C_{a, b} r\right)}{\mu B\left(b, c_{a, b} r\right)}
$$


for $0<r<r_{a, b}$, it follows that

$$
\lim _{r \downarrow 0} \frac{\mu(A(a, b, r) \backslash B)}{\mu A(a, b, r)}=0, \quad \text { whence } \lim _{r \downarrow 0} \frac{\mu(B \cap A(a, b, r))}{\mu A(a, b, r)}=1 .
$$

Put $L=\lim \sup _{r \downarrow 0} \nu A(a, b, r) / \nu B(a, r)$ and choose $u>1$ with $u^{4} s<t$. Then there is $r>0$ such that

$$
\begin{aligned}
\nu B(a, r) & <u \nu(A \cap B(a, r)), \mu B(a, r)<\mu L^{-1} \mu A(a, b, r), \\
\mu A(a, b, r) & \leqslant u \mu(B \cap A(a, b, r)), \nu A(a, b, r)<u L \nu B(a, r) .
\end{aligned}
$$

Then

$$
\begin{aligned}
\nu B(a, r) & \leqslant u \nu(A \cap B(a, r))=u \int_{A \cap B(a, r)} f d \mu<u s \mu B(a, r) \\
& \leqslant u^{2} s L^{-1} \mu A(a, b, r)<u^{3} s L^{-1} \mu(B \cap A(a, b, r)) \\
& \leqslant u^{3} s t^{-1} L^{-1} \int_{B \cap A(a, b, r)} f d \mu=u^{3} s t^{-1} L^{-1} \nu(B \cap A(a, b, r)) \\
& \leqslant u^{4} s t^{-1} \nu B(a, r)<\nu B(a, r) .
\end{aligned}
$$

This contradiction proves that there is a constant $c$ such that $f(x)=c$ for $\mu$ a.a. $x \in X$. Hence $\nu=c \mu$.

3.5. Corollary. Let $\mu$ and $\nu$ be Borel regular measures on $X$. If there exist $g \in H^{*}$ and $h \in H$ such that $0<D_{g}(\mu, x)=D_{h}(\nu, x)<\infty$ for $x \in X$, then there is a constant $c$ such that $\nu=c \mu$.

Proof. We may choose $A(x, y, r)=B(y, r)$.

The following corollary to Theorem 3.4 contains a theorem of Engle [2], which earlier was proved in a weaker form by Mickle and Radó [5]. Let $\mathcal{H}$ be a family of homeomorphisms of $X$. A measure $\mu$ on $X$ is said to be $\mathcal{X}$-invariant if $\mu(f(E))=$ $\mu(E)$ for all $E \subset X$ and $f \in \mathcal{H}$, and if $0<\mu(G)<\infty$ for some open set $G \subset X$.

3.6. Corollary. Suppose that $\mathcal{H}$ is a family of homeomorphisms of $X$ and that for all $x, y \in X$ there are $0<c_{x, y}<C_{x, y}<\infty, r_{x, y}>0$ and $h_{x, y} \in \mathcal{H}$ such that for $0<r<r_{x, y}$,

$$
B\left(y, c_{x, y} r\right) \subset h_{x, y}(B(x, r)) \subset B\left(y, C_{x y} r\right)
$$

If $\mu$ and $\nu$ are $\mathcal{H}$-invariant Borel regular measures on $X$ and if there is $x_{0} \in X$ such that $\lim \sup _{r \downarrow 0} \mu B\left(x_{0}, 5 r\right) / \mu B\left(x_{0}, r\right)<\infty$, then there is a constant $c$ such that $\nu=c \mu$.

Proof. We may take $A(x, y, r)=h_{x, y}(B(x, r))$, since then $\mu A(x, y, r)=\mu B(x, r)$ and $\nu A(x, y, r)=\nu B(x, r)$.

4. The infinite-dimensional case. We now turn to treat the infinite-dimensional case, where no $5 r$-condition is assumed. The following density lemma is a special case of the differentiation theorems of [4], but we include its proof for completeness. 
4.1. Lemma. Suppose that $\mu$ is a Borel regular measure on $X, g \in H$ and $E$ is a $\mu$ measurable subset of $X$. If $\mu B(x, r)<g(r)$ for $\mu$ a.a. $x \in X$ and $r>0$, then for any $\mu$ measurable set $B \subset E$ with $\mu(B)<\infty$,

$$
\lim _{r \downarrow 0} \int_{B} \frac{\mu(B(x, r) \backslash E)}{g(r)} d \mu x=0 .
$$

Proof. Let $\varepsilon>0$. Since $X$ is separable and every ball has finite $\mu$ measure, there is an open set $G$, by $2.2(2)$, such that $B \subset G$ and $\mu(G \backslash B)<\varepsilon$. Letting

$$
G_{r}=\{x: \operatorname{dist}(x, X \backslash G)>r\} \text { for } r>0,
$$

we have $\lim _{r \downarrow 0} \mu\left(G \backslash G_{r}\right)=0$, and we choose $r_{0}>0$ such that $\mu\left(G \backslash G_{r}\right)<\varepsilon$ for $0<r<r_{0}$. By 2.2(1) there is a closed set $F \subset B \cap G_{r_{0}}$ such that $\mu\left(B \cap G_{r_{0}} \backslash F\right)<$ $\varepsilon$. Then

$$
\begin{aligned}
& F_{r}=\{x: \operatorname{dist}(x, F)<r\} \subset G \text { for } 0<r<r_{0} \\
& \mu(B \backslash F)<\mu\left(G \backslash G_{r_{0}}\right)+\mu\left(B \cap G_{r_{0}} \backslash F\right)<2 \varepsilon
\end{aligned}
$$

and

$$
\mu\left(F_{r_{0}} \backslash F\right)<\mu(G \backslash B)+\mu(B \backslash F)<3 \varepsilon .
$$

Applying Fubini's theorem to the characteristic function of the set $\{(x, y): d(x, y)$ $<r$, we obtain for $0<r<r_{0}$,

$$
\begin{aligned}
g(r)^{-1} \int_{B} \mu(B(x, r) \backslash E) d \mu x & <g(r)^{-1} \int_{F} \mu(B(x, r) \backslash F) d \mu x+2 \varepsilon \\
& =g(r)^{-1} \int_{F} \mu\left(B(x, r) \cap F_{r} \backslash F\right) d \mu x+2 \varepsilon \\
& =g(r)^{-1} \int_{F_{r} \backslash F} \mu(B(x, r) \cap F) d \mu x+2 \varepsilon<5 \varepsilon .
\end{aligned}
$$

This proves the lemma.

Since mean convergence implies pointwise convergence a.e. for a subsequence we obtain the following

4.2. Corollary. If in addition to the hypothesis of Theorem $4.1, D_{B}(\mu, x)>0$ for $\mu$ a.a. $x \in E$, then every sequence $\left(r_{i}\right)$ of positive numbers tending to zero has $a$ subsequence $\left(r_{i}\right)$ such that

$$
\lim _{j \rightarrow \infty} \frac{\mu\left(B\left(x, r_{i j}\right) \backslash E\right)}{\mu B\left(x, r_{i j}\right)}=0 \text { and } \lim _{j \rightarrow \infty} \frac{\mu\left(E \cap B\left(x, r_{i j}\right)\right)}{\mu B\left(x, r_{i j}\right)}=1
$$

for $\mu$ a.a. $x \in E$.

The proof of the following theorem resembles a proof given by Christensen [1].

4.3. TheOREM. Suppose that $\mu$ and $\nu$ are Borel regular measures on $X$ and $g, h \in H . I f$

(1) $\underline{D}_{s}(\mu, x)>0$ and $\mu B(x, r)<g(r)$ for $x \in X$ and $r>0$ and

(2) $\vec{D}_{h}(\nu, x)>0$ and $\nu B(x, r)<h(r)$ for $x \in X$ and $r>0$,

then 3.3(3) holds and $\mu$ and $\nu$ are mutually absolutely continuous. 
Proof. Suppose that $A$ is a Borel subset of $X$ with $\mu(A)=0$ and $G$ is an open set with $A \subset G$ and $\mu(G)<\infty$. As in the proof of 4.1, Fubini's theorem gives for all $r>0$,

$$
\int_{G} \mu(G \cap B(x, r)) d \nu x=\int_{G} \nu(G \cap B(x, r)) d \mu x<h(r) \mu(G) .
$$

Using the fact that $G$ is open and Fatou's lemma, we obtain

$$
\begin{aligned}
\int_{A} \underline{D}_{g}(\mu, x) d \nu x & <\int_{G} \lim _{r \downarrow 0} \inf \frac{\mu(G \cap B(x, r))}{g(r)} d \nu x \\
& <\lim _{r \downarrow 0} \inf _{G} \frac{\mu(G \cap B(x, r))}{g(r)} d \nu x<\left(\liminf _{r \downarrow 0} \frac{h(r)}{g(r)}\right) \mu(G) .
\end{aligned}
$$

Since $\underline{D}_{s}(\mu, x)>0$ for $x \in G$ by (1) and $\nu(G)>0$ by (2), we have $\lim \inf _{r \downarrow 0} h(r) / g(r)>0$, whence $\lim \sup _{r \downarrow 0} g(r) / h(r)<\infty$. In the same way $\lim \inf _{r \downarrow 0} g(r) / h(r)>0$. For any $\varepsilon>0$ we can take $G$ such that $\mu(G)<\varepsilon$ by $2.2(2)$. It follows that $\int_{A} \underline{D}_{g}(\mu, x) d \nu x=0$, which implies $\nu(A)=0$. This proves that $\nu$ is absolutely continuous with respect to $\mu$. In the same way one shows that $\mu$ is absolutely continuous with respect to $\nu$.

4.4. TheOREM. Let $\mu$ and $\nu$ be Borel regular measures on $X$. Suppose that there is $r_{0}>0$ and that for each $x \in X$ there are $0<\lambda_{x}<\infty$ and functions $\varphi_{x}, \psi_{x}$ : $\left(0, r_{0}\right) \rightarrow(0, \infty)$ such that $\varphi_{x}(r)<r<\psi_{x}(r)$ for $0<r<r_{0}$ and

$$
\mu B\left(y, \psi_{x}(r)\right)<\lambda_{x} \mu B\left(y, \varphi_{x}(r)\right), \quad \nu B\left(y, \psi_{x}(r)\right)<\lambda_{x} \nu B\left(y, \varphi_{x}(r)\right)
$$

for $y \in X, 0<r<r_{0}$. Suppose also that there is $x_{0} \in X$ and that for any $x, y \in X$, $0<r<r_{0}$ there is a Borel set $A(x, y, r)$ such that

$$
\begin{gathered}
B\left(y, \varphi_{x}(r)\right) \subset A(x, y, r) \subset B\left(y, \psi_{x}(r)\right), \quad \liminf _{r \downarrow 0} \frac{\nu A\left(x_{0}, x, r\right)}{\nu B\left(x_{0}, r\right)}>0, \\
\mu A\left(x_{0}, x, r\right)<\lambda_{x_{0}} \mu B\left(x_{0}, r\right), \quad \nu A\left(x_{0}, x, r\right)<\lambda_{x_{0}} \nu B\left(x_{0}, r\right)
\end{gathered}
$$

and

$$
\underset{r \downarrow 0}{\lim \sup } \frac{\nu A(x, y, r)}{\nu B(x, r)}<\liminf _{r \downarrow 0} \frac{\mu A(x, y, r)}{\mu B(x, r)} .
$$

Then there is a constant $c$ such that $\nu=c \mu$.

Proof. The proof is similar to that of Theorem 3.4. Define $g(r)=\mu B\left(x_{0}, r\right)$ and $h(r)=\nu B\left(x_{0}, r\right)$. Then

$$
\frac{\mu B(x, r)}{g(r)}>\frac{\mu B\left(x, \varphi_{x_{0}}(r)\right)}{\mu B\left(x, \psi_{x_{0}}(r)\right)} \cdot \frac{\mu A\left(x_{0}, x, r\right)}{\mu B\left(x_{0}, r\right)}>\lambda_{x}^{-1} \frac{\mu A\left(x_{0}, x, r\right)}{\mu B\left(x_{00} r\right)},
$$

whence $\lim \inf _{r \downarrow 0} \mu B(x, r) / g(r)>0$, and

$$
\mu B(x, r)<\lambda_{x_{0}} \mu B\left(x, \varphi_{x_{0}}(r)\right)<\lambda_{x_{0}} \mu A\left(x_{0}, x, r\right)<\lambda_{x_{0}}^{2} g(r) .
$$

Similar inequalities for $\nu$ and $h$ show that 4.1-4.3 are applicable. By Theorem 4.3, $\mu$ and $\nu$ are mutually absolutely continuous and the Radon-Nikodým theorem yields a positive and finite Borel function $f$ on $X$ such that $\nu(A)=\int_{A} f d \mu$ for all Borel sets 
$A \subset X$. If $f$ is not constant $\mu$ a.e., there are $0<s<t<\infty$ such that the sets $A=\{x: f(x)<s\}, B=\{x: f(x)>t\}$ have positive $\mu$ and $\nu$ measure. Then by Corollary 4.2 there are $a \in A$ and a sequence $\left(r_{i}\right)$ tending to 0 such that

$$
\lim _{i \rightarrow \infty} \frac{\nu\left(A \cap B\left(a, r_{i}\right)\right)}{\nu B\left(a, r_{i}\right)}=1 \text {. }
$$

Let $s_{i}=\varphi_{a}\left(r_{i}\right)$ and $t_{i}=\psi_{a}\left(r_{i}\right)$. Then by Theorem 4.1 there is $b \in B$ such that

$$
\liminf _{i \rightarrow \infty} \frac{\mu\left(B\left(b, t_{i}\right) \backslash B\right)}{g\left(t_{i}\right)}=0 .
$$

Denote $A_{i}=A\left(a, b, r_{i}\right)$. Since for large $i$,

$$
\frac{\mu\left(A_{i} \backslash B\right)}{\mu\left(A_{i}\right)}<\frac{\mu\left(B\left(b, t_{i}\right) \backslash B\right)}{g\left(t_{i}\right)} \cdot \frac{g\left(s_{i}\right)}{\mu B\left(b, s_{i}\right)} \frac{g\left(t_{i}\right)}{g\left(s_{i}\right)},
$$

$g\left(t_{i}\right)<\lambda_{a} g\left(s_{i}\right)$ and $\lim \inf _{i \rightarrow \infty} \mu B\left(b, s_{i}\right) / g\left(s_{i}\right)>0$, we obtain

$$
\liminf _{i \rightarrow \infty} \frac{\mu\left(A_{i} \backslash B\right)}{\mu\left(A_{i}\right)}=0, \text { which gives } \limsup _{i \rightarrow \infty} \frac{\mu\left(A_{i} \cap B\right)}{\mu\left(A_{i}\right)}=1 .
$$

Put $L=\lim \sup _{i \rightarrow \infty} \nu\left(A_{i}\right) / \nu B\left(a, r_{i}\right)$ and choose $u>1$ such that $u^{4} s<t$. Then there is $i$ such that

$$
\begin{aligned}
\nu B\left(a, r_{i}\right) & <u \nu\left(A \cap B\left(a, r_{i}\right)\right)=u \int_{A \cap B\left(a, r_{i}\right)} f d \mu<u s \mu B\left(a, r_{i}\right) \\
& <u^{2} s L^{-1} \mu\left(A_{i}\right)<u^{3} s L^{-1} \mu\left(A_{i} \cap B\right)<u^{3} s t^{-1} L^{-1} \int_{A_{i} \cap B} f d \mu \\
& <u^{3} s t^{-1} L^{-1} \nu\left(A_{i}\right)<u^{4} s t^{-1} \nu B\left(a, r_{i}\right)<\nu B\left(a, r_{i}\right) .
\end{aligned}
$$

This contradiction proves that $f$ is constant $\mu$ a.e., and the theorem follows.

4.5. Corollary. Let $\mu$ and $\nu$ be Borel regular measures on $X$. If there exist $g, h \in H$ such that for all $x \in X, \mu B(x, r)<g(r), \nu B(x, r)<h(r)$ for $r>0$ and $0<D_{g}(\mu, x)=D_{h}(\nu, x)$, then there is a constant $c$ such that $\nu=c \mu$.

Proof. We may take $\varphi_{x}(r)=\psi_{x}(r)=r, A(x, y, r)=B(y, r)$, because then there are $x_{0} \in X, C<\infty$ and $r_{0}>0$ such that $\mu B(x, r)<g(r)<C \mu B\left(x_{0}, r\right)$ and $\nu B(x, r)<h(r)<C \nu B\left(x_{0}, r\right)$ for $x \in X, 0<r<r_{0}$.

4.6. Corollary. Let $\mu$ and $\nu$ be Borel regular measures on $X$ and $\mathcal{H}$ a family of homeomorphisms of $X$. Suppose that there is $r_{0}>0$ and that for each $x \in X$ there are $0<\lambda_{x}<\infty$ and functions $\varphi_{x}, \psi_{x}:\left(0, r_{0}\right) \rightarrow(0, \infty)$ such that $\varphi_{x}(r)<r<\psi_{x}(r)$ for $0<r<r_{0}$ and

$$
\mu B\left(y, \psi_{x}(r)\right)<\lambda_{x} \mu B\left(y, \varphi_{x}(r)\right), \quad \nu B\left(y, \psi_{x}(r)\right)<\lambda_{x} \nu B\left(y, \varphi_{x}(r)\right)
$$

for $y \in X$ and $r>0$. Suppose also that for all $x, y \in X$ there is $h_{x, y} \in \mathcal{H}$ such that

$$
B\left(y, \varphi_{x}(r)\right) \subset h_{x, y}(B(x, r)) \subset B\left(y, \psi_{x}(r)\right)
$$

for $0<r<r_{0}$. If $\mu$ and $\nu$ are $\mathcal{H}$-invariant, then there is a constant $c$ such that $\nu=c \mu$. 
4.7. Remark. The following example, which was constructed in [4], shows that the 5r-condition in 3.1-3.5 cannot be dropped:

There exist a compact metric space $X$, a Borel regular measure $\mu$ on $X, g \in H$ and a Borel set $E \subset X$ such that $\mu(E)<\mu(X)=1$ and $D_{g}(\mu, x)=D_{g}(\mu\llcorner E, x)=$ 1 for $\mu$ a.a. $x \in X$. Here $\mu$ is not absolutely continuous with respect to $\mu L E$.

\section{REFERENCES}

1. J. P. R. Christensen, On some measures analogous to Haar measure, Math. Scand. 26 (1970), 103-106.

2. J. A. Engle, A 5r-uniqueness theorem, Trans. Amer. Math. Soc. 201 (1975), 89-105.

3. H. Federer, Geometric measure theory, Springer-Verlag, Berlin and New York, 1969.

4. P. Mattila, Differentiation of measures on uniform spaces, Proc. Measure Theory Conf. (Oberwolfach, 1979), Springer-Verlag, Berlin and New York (to appear).

5. E. J. Mickle and T. Radó, A uniqueness theorem for Haar measure, Trans. Amer. Math. Soc. 93 (1959), 492-508.

Department of Mathematics, University of Helsinka, Hallituskatu 15, 00100 Helsinki 10, FinLAND 